Article

\title{
A Novel Three-Dimensional Wound Healing Model
}

\section{Zhuo J. Chen ${ }^{1}$, Jessica P. Yang ${ }^{1}$, Benjamin M. Wu ${ }^{1,2}$ and Bill Tawil 1,*}

1 Department of Bioengineering, UCLA School of Engineering, 420 Westwood Plaza, Los Angeles, CA 90095-1600, USA; E-Mails: zhuojc787@engineering.ucla.edu (Z.J.C.); jpwyang@gmail.com (J.P.Y.); benwu@ucla.edu (B.M.W.)

2 Department of Materials Science and Engineering, UCLA School of Engineering, 420 Westwood Plaza, Los Angeles, CA 90095-1600, USA

* Author to whom correspondence should be addressed; E-Mail: btawil@seas.ucla.edu; Tel.: +1-310-794-6140; Fax: +1-310-794-5956.

External Editor: Robin Muise-Helmericks

Received: 13 October 2014; in revised form: 7 November 2014 / Accepted: 10 December 2014 / Published: 19 December 2014

\begin{abstract}
Wound healing is a well-orchestrated process, with various cells and growth factors coming into the wound bed at a specific time to influence the healing. Understanding the wound healing process is essential to generating wound healing products that help with hard-to-heal acute wounds and chronic wounds. The 2D scratch assay whereby a wound is created by scratching a confluent layer of cells on a 2D substrate is well established and used extensively but it has a major limitation - it lacks the complexity of the 3D wound healing environment. Established 3D wound healing models also have many limitations. In this paper, we present a novel 3D wound healing model that closely mimics the skin wound environment to study the cell migration of fibroblasts and keratinocytes. Three major components that exist in the wound environment are introduced in this new model: collagen, fibrin, and human foreskin fibroblasts. The novel 3D model consists of a defect, representing the actual wound, created by using a biopsy punch in a 3D collagen construct. The defect is then filled with collagen or with various solutions of fibrinogen and thrombin that polymerize into a 3D fibrin clot. Fibroblasts are then added on top of the collagen and their migration into the fibrin - or collagen - filled defect is followed for nine days. Our data clearly shows that fibroblasts migrate on both collagen and fibrin defects, though slightly
\end{abstract}


faster on collagen defects than on fibrin defects. This paper shows the visibility of the model by introducing a defect filled with fibrin in a 3D collagen construct, thus mimicking a wound. Ongoing work examines keratinocyte migration on the defects of a 3D construct, which consists of collagen-containing fibroblasts. The model is also used to determine the effects of various growth factors, delivered in the wound defects, on fibroblasts' and keratinocytes' migration into the defects. Thus this novel $3 \mathrm{D}$ wound healing model provides a more complex wound healing assay than existing wound models.

Keywords: fibrin; 3D construct; cell migration

\section{Introduction}

Wound healing is a complex process that consists of four main stages: hemostasis, inflammation, proliferation, and remodeling of the tissue [6,21]. In the initial stage of wound healing, a fibrin clot is formed at the site of the wound shortly following injury [6,21]. Fibrinogen is cleaved into fibrin monomers by thrombin, and the peptide monomers are polymerized by Factor XIII [21]. The resultant fibrin acts as a scaffold for various cells to move in and out of the wound bed [21]. Five to seven days after the initial injury, fibroblasts migrate to the wound site, secreting new collagen, and keratinocytes migrate from the wound edge and form a thin epithelial cell layer to close the wound [6,21]. Various growth factors secreted by invading cells such as macrophages, fibroblasts, and keratinocytes play an essential role during this process [6,21]. Examples of these growth factors include epidermal growth factor (EGF), vascular endothelial growth factor (VEGF), fibroblast growth factor (FGF), and platelet-derived growth factor (PDGF) $[1,2,10,14]$.

In the United States, chronic wounds cost \$20-25 billion a year, and acute or traumatic wounds add another \$7-10 billion annually. There are highly developed wound products including wound dressings (alginates hydrocolloids and hydrogels), skin substitutes, and growth factor based products that are used to treat chronic wounds [21]. It is known that these products do not work well on all patients, suggesting a lack of understanding of the wound healing process, especially in chronic wounds. To that extent, it is very important to establish a 3D wound healing model that helps with better understanding wound healing in general and cell migration specifically. Existing wound healing models range from simple 2D in vitro models to $3 \mathrm{D}$ in vivo models [3]. In the well-established 2D scratch assay, a wound is created by scratching a confluent layer of cells seeded on a substrate and cell migration into the scratch (wound) is followed [20]. A major limitation of this model is that it lacks the complexity of the wound bed microenvironment. More advanced 3D wound healing models have been established over the last few years. For example, in a wound healing model by Karamichos et al., 2009 [12], fibroblasts are embedded in a 3D collagen construct and the cell migration is followed from the denser collagen matrix into a surrounding matrix. The limitations of this model include the use of only one type of ECM protein and one cell type. An improved 3D in vitro model is the "human skin equivalents" [3,22]; it contains a stratified layer of keratinocytes and keeps the surface at an air-liquid interface. In this model, the wound closes in 48-72 h, which is comparable to the in vivo environment [3,22]. A third 3D model is an example of a 3D in vivo model where skin equivalents are cultured with keratinocytes and then grafted into 
mice [9]. Our novel 3D model presented in this study has many advantages including the ability to use more than one substrate and more than one cell type in a 3D construct that mimics the wound bed. This work is based on the extensive work that this lab published over the last 10 years studying the proliferation, migration, and behavior of various cell types including fibroblasts, keratinocytes, monocytes, and mesenchymal stem cells in a 3D fibrin construct $[4,7,8,11,15,16,19]$.

In this paper, we present a novel 3D wound healing model that better resembles the wound bed during the wound healing process. The novelty of this model is the ability to cointroduce multiple cell types and many growth factors and be able to measure the cell migration. This initial paper introduces this new 3D wound healing model. We used fibroblasts as an example to show the complexity and the potential of the model. Ongoing work includes cointroducing fibroblasts in the $3 \mathrm{D}$ collagen construct while adding keratinocytes on the top and following the migration of both cells in the defect. We are also using the model to examine the effect of various growth factors introduced in the defect on cell migration. The model could also be used to study the effect of different drugs on cell migration during the wound healing process.

\section{Materials and Methods}

\subsection{Cells}

Human foreskin fibroblast (HFFs) lines from ATCC (Manassas, VA, USA) were cultured in Dulbecco's modified Eagle's Medium (DMEM) from CellGro (Manassas, VA, USA) with $4.5 \mathrm{mg} / \mathrm{mL}$ glucose, $10 \%$ fetal bovine serum, and 5\% penicillin/streptomycin. Cells were maintained in an incubator at $37{ }^{\circ} \mathrm{C}$ and $5 \% \mathrm{CO}_{2}$. Human epithelial keratinocytes (HEK001, ATCC CRL-2404, Manassas, VA, USA) were cultured in keratinocyte serum-free media (GIBCO) in $5 \% \mathrm{CO}_{2}$ at $37{ }^{\circ} \mathrm{C}$. The medium was changed every three days, and cells were passaged to new flasks upon reaching confluence. Passage 6-10 keratinocytes were used.

\subsection{D Scratch Wound Assay}

One milliliter of protein-containing solution (fibrinogen or collagen) was pipetted into a 12-well plate. The well plate (polysterene) was used as a comparative control. After a one-hour incubation to allow proteins to adsorb to the surface, the remaining solution was removed and the surface was washed twice with DPBS. Eighty thousand cells $/ \mathrm{cm}^{2}$ were seeded in each well. A reference point was drawn on the bottom of each well. The plate was incubated overnight to allow the cells to adhere to the substrate.

The next day, a pipette tip was used to scratch the confluent cell layer. A Leica DM IRB bright-field microscope was used to image each scratch wound at the reference point. This process was repeated at hourly time points to monitor the cell migration to close the wound area. 


\subsection{Preparation of the $3 D$ Model}

Biopsy Punch Fabrication

Seven hundred microliter $2.5 \mathrm{mg} / \mathrm{mL}$ Purecol (Advanced Biomatrix, San Diego, CA, USA) collagen gels were formed in a 12 -well plate. $\mathrm{NaOH}(0.1 \mathrm{~N})$ was added to the collagen and $1 \times \mathrm{PBS}$ to neutralize and polymerize the collagen. The well plate was placed in the incubator at $37^{\circ} \mathrm{C}$ for an hour to allow the collagen to fully polymerize. HFFs were trypsinized using TrypLE Select (Gibco). A specific number of HFFs were isolated to give a final concentration of $3 \times 10^{5}$ cells/gel and stained with Vybrant DiO (Invitrogen, Carlsbad, CA, USA). After staining, the cells were washed three times, then seeded onto the collagen gel. After $3 \mathrm{~h}$, a $2 \mathrm{~mm}$ biopsy punch attached to a vacuum line was used to punch regions out of the collagen gel. These defects were then filled with $2.5 \mathrm{mg} / \mathrm{mL}$ collagen or $10 \mathrm{mg} / \mathrm{mL}$ purified fibrinogen (Enzyme Research, South Bend, IN, USA) with $10 \mathrm{IU} / \mathrm{mL}$ thrombin (Gibco, Carlsabad, CA, USA). Once the defects were polymerized, $1 \mathrm{~mL}$ of media was added to each sample well.

\subsection{Preparing Conditioned Media}

For each 3D model, a 2D layer of confluent HFFs was seeded in a separate well plate. We prepared the conditioned media in a separate well plate; the number of wells needed corresponded to the total sample wells containing the 3D model. For the scratch-conditioned media, fresh medium was added, and the 2D layer of cells was scratched with a pipette tip $24 \mathrm{~h}$ prior to the time point. For the confluent conditioned media, fresh medium was supplied to a confluent 2D layer of HFFs $24 \mathrm{~h}$ prior to the time point. After each time point, the medium from the 2D layer was removed, spun down, and added to the 3D construct. For the control, fresh medium was added.

\subsection{Measuring Cell Migration}

Prior to imaging, the gel surface was gently rinsed three times with medium. The construct was imaged using a Nikon Eclipse Ti fluorescent microscope at day 0, 3, 6, and 9. The FITC filter was used to image the HFFs. The images were further analyzed using ImageJ software to quantify the distance between migrated cells and the wound edge. Day 0 was treated as our reference, since the defect area shrunk as HFFs moved toward the center of the defect, and the migration rate was obtained by plotting the data points from ImageJ.

\subsection{Statistical Analysis}

Data were presented as the mean \pm standard error, with $n=3$ (triplicate constructs per condition, repeated three times). Data were assessed using Student's $t$-test, with $p<0.05$ considered significant.

\section{Results}

\subsection{Cell Migration in 2D Scratch Assays}

In this experiment, we created a scratch in a confluent layer of fibroblasts seeded on collagen, fibrin, or polystyrene and measured the fibroblasts migration over two days. Visual analysis (Figure 2) showed 
that fibroblasts migrated into the wound within $31 \mathrm{~h}$ on all three substrates. The migration rate was calculated to be of $630.30 \mu \mathrm{m} /$ day. After analysis of the distance migrated and looking at a percent of total distance migrated, there was not a significant difference among the different substrates (Figure 3).

Figure 2. Two-dimensional scratch wound assay. Fibroblast migration on (A) $3.0 \mathrm{mg} / \mathrm{mL}$ collagen, (B) $5 \mathrm{mg} / \mathrm{mL}$ plasminogen depleted fibrin, or (C) polysterene substrates. The arrows on the right in Figure 1 point to the defects; there are four of them in each 3D construct. Images were taken at $2.5 \times$ magnification using a Leica DM IRB.

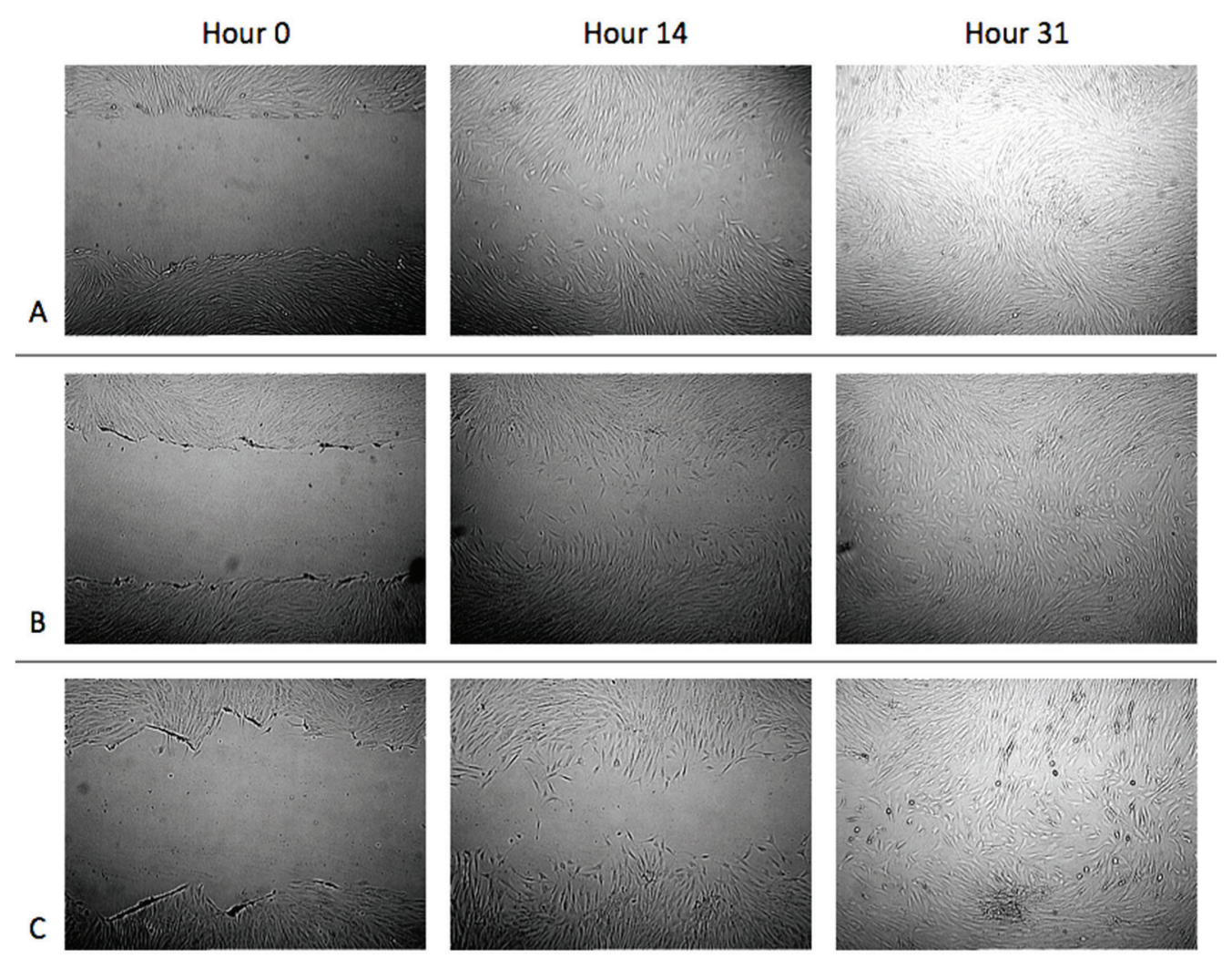

Figure 3. Two-dimensional scratch wound assay. Percent of total distance migrated. Distance migrated measured in ImageJ as the distance between the two leading edges subtracted from the total distance at hour 0 .

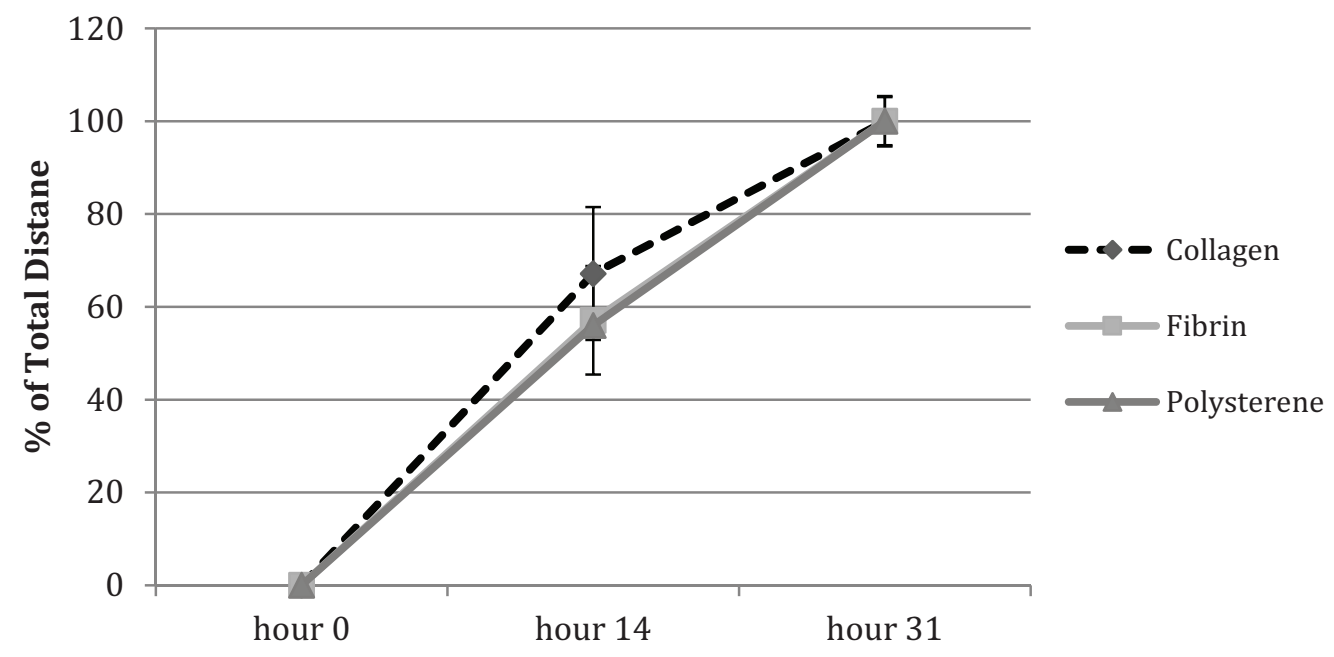




\subsection{Cell Migration on 3D Models}

\subsubsection{HFF Migration from Collagen to Collagen}

The new 3D wound healing model consists of fibroblasts added on the top of a 3D collagen construct with four biopsies (wounds) that are equally separated from each other and from the edge of the well. The generated wounds are then filled with either collagen or fibrin. ImageJ software is used to measure the distance from the wound edge to the leading edge of migrated cells. Pixel measurements are converted to micrometers by using a hemocytometer for scale.

For small wound defects, fibroblasts migrated over half of the defect by day 3 , and completely covered the defect by day 6 in most trials. For lager wound defects, although fibroblasts did not cover the entire defect as fast as those with a smaller wound area, the migration rate was similar. Thus, the size of the defect did not have a noticeable effect on the cell migration rate. Quantification of the images (Figure 4) illustrated that the bulk of the migration has occurred by day 6 . This corresponded with an overall migration rate of $249.09 \mu \mathrm{m} /$ day.

Next, we examined the effect of using a conditioned medium from $2 \mathrm{D}$ scratched assays to determine if the released growth factors and chemoattractants due to the scratch (wound) affect cell migration in the new 3D wound model. To that extent, we used three different sources of media: first, a fresh media added directly to the 3D construct; secondly, the source was a conditioned medium from the unscratched 2D assay; and, finally, a conditioned medium was taken from the scratched 2D assay. Comparing the rate of migration among the three conditions, we found that there was a slightly higher rate of migration with conditioned media but not statistically significant (Figures 4 and 5).

Figure 4. Three-dimensional wound migration assay. Fibroblast migration from collagen to a collagen defect with (A) control medium, (B) conditioned medium from a scratched 2D layer of HFFs, or (C) conditioned medium from a non-scratch confluent 2D layer of HFFs. Migration measured using ImageJ as distance from wound edge to edge of migrated cells. Cells began to infiltrate the wound area by day 3 and cover the wound by day 6 for defects that had a similar size, shown here. For larger defect areas, cells completely covered the wound by day 9 in the majority of cases. Images taken at $2.5 \times$ magnification.

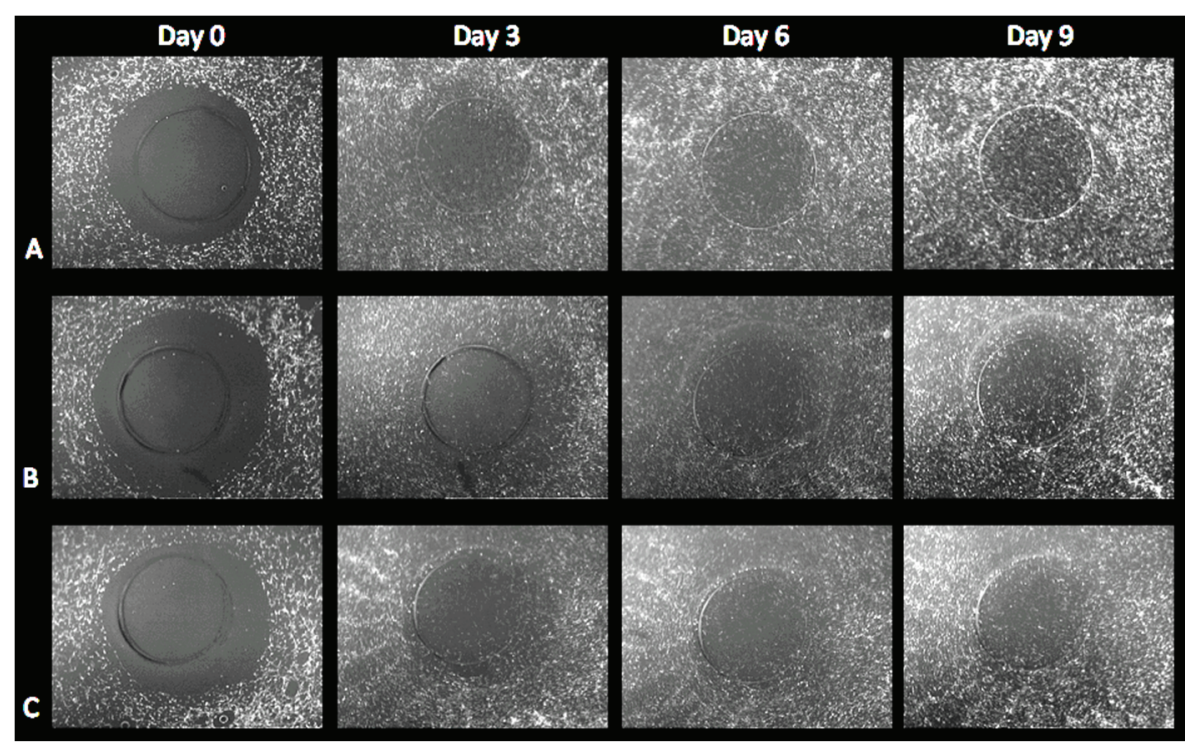


Figure 5. Three-dimensional wound migration assay. Fibroblast migration from collagen to a collagen defect with two medium conditions (non-scratch and scratch). Distance migrated measured in Image J as the distance between the wound edge and the leading edge of cell migration.

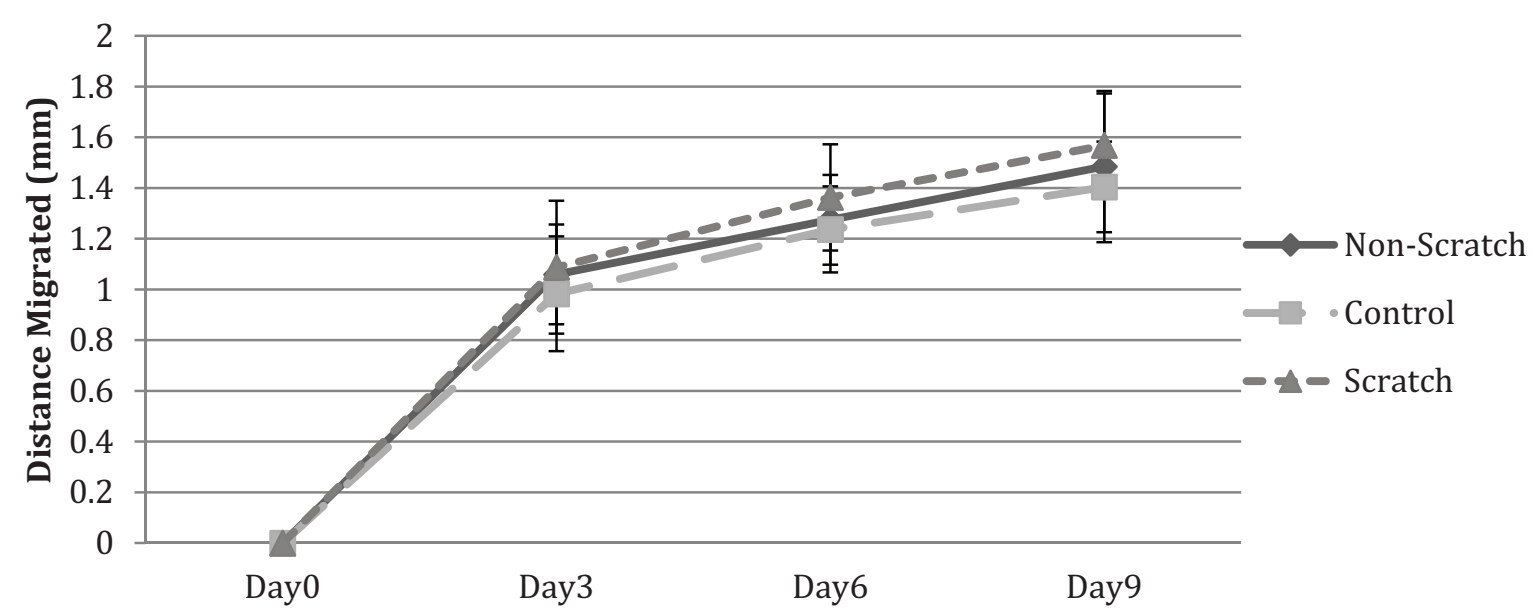

Figure 6. Three-dimensional wound migration assay. Fibroblast migration from collagen to a fibrin defect with (A) control medium, (B) conditioned medium from a scratched 2D layer of HFFs, or (C) conditioned medium from a non-scratch confluent 2D layer of HFFs. Migration measured using ImageJ as distance from wound edge to edge of migrated cells. Cells began to infiltrate the wound area by day 3 and cover the wound by day 6 for defects that had a similar size, shown here. For larger defect areas, cells completely covered the wound by day 9 in the majority of cases. Images taken at $2.5 \times$ magnification.

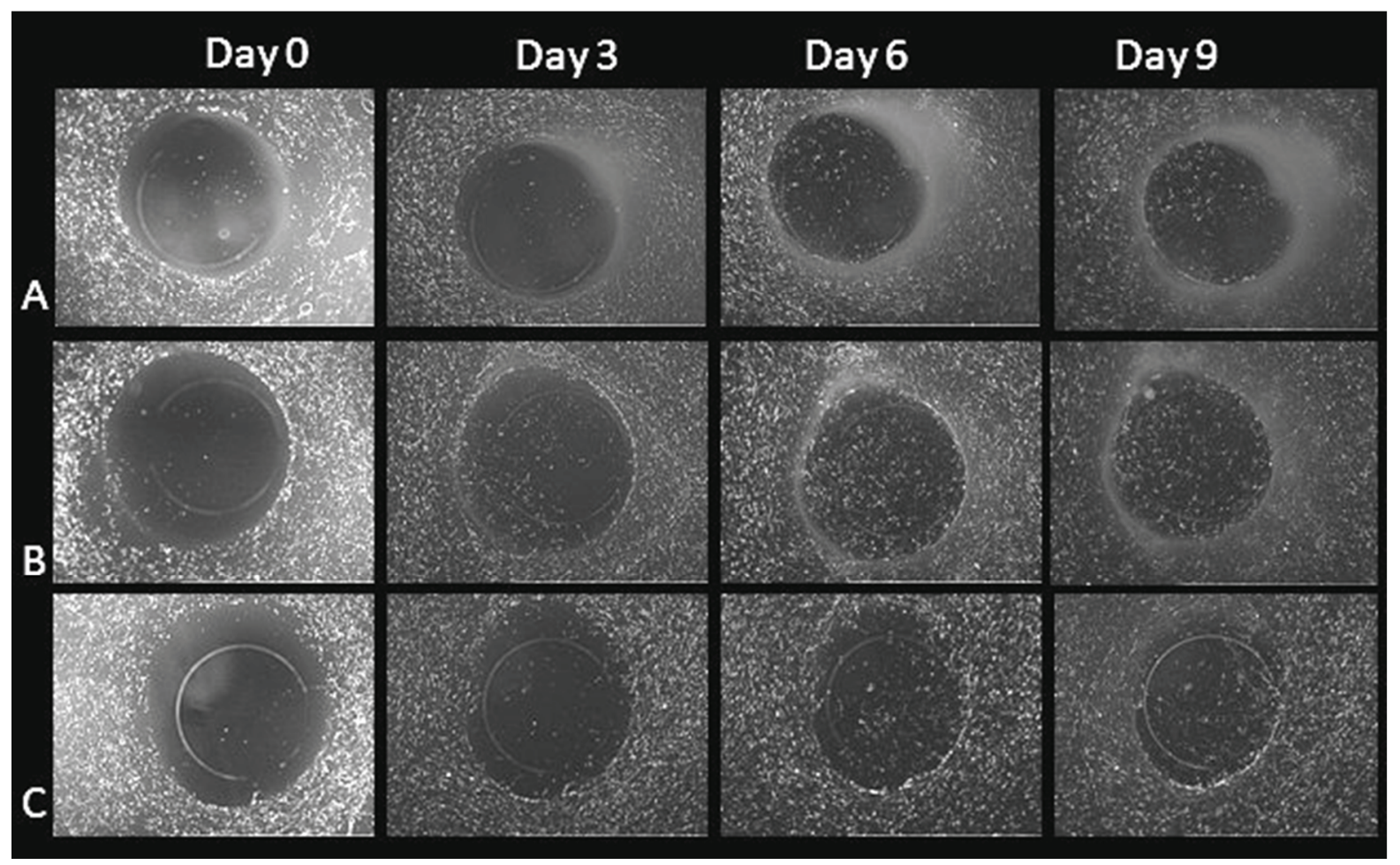




\subsubsection{HFF Migration from Collagen to Fibrin}

We filled in fibrin in the defects instead of collagen in a 3D collagen construct. The rate of migration is determined by the same method as mentioned above for the collagen-to-collagen model. In 3D constructs with small wound size defects, fibroblasts covered the entire defect by day 6 , and there were not any significant differences in migration rate among fresh medium and conditioned medium from scratched or unscratched 2D plates (Figure 6). The migration rate of fibroblasts from collagen to fibrin was $144 \mu \mathrm{m} /$ day on average (Figure 7). In other words, changing the defect filled in from collagen to fibrin, which more closely resembled the actual wound environment, the distance of migration observed from the collagen to fibrin model was slightly less than those from the collagen to collagen model, but closer to the in vivo studies.

Figure 7. Three-dimensional wound migration assay. Fibroblast migration from collagen to a fibrin defect with two medium conditions (non-scratch and scratch). Distance migrated measured in Image J as the distance between the wound edge and the leading edge of cell migration.

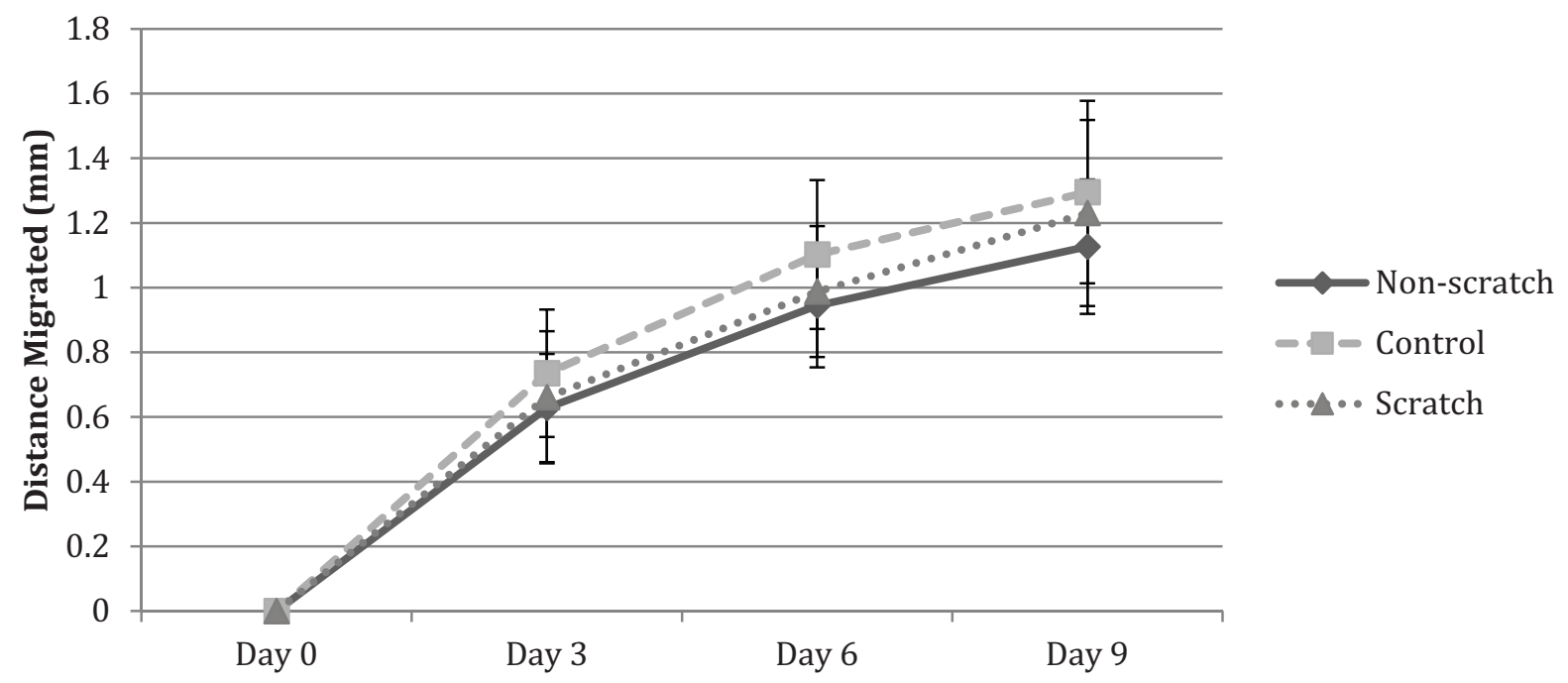

\subsubsection{HFF Migration: Biopsy Punch vs. Mold Model}

We have also tested a different approach to create a defect. We used a mold to fabricate the wound defects, which compressed the sample to the shape of the mold. We did not continue with the mold model since cell migration was not observed using the mold constructs (Figure 8). Two types of cells, fibroblasts and human epidermal keratinocytes, were introduced separately into the model, but both ended up with similar results. Therefore, we discontinued the mold model approach. 
Figure 8. Three-dimensional wound migration assay with the mold model. Migration of human epidermal keratinocytes from collagen construct to various defects was investigated. No migration was observed. Images taken at $2.5 \times$ magnification.

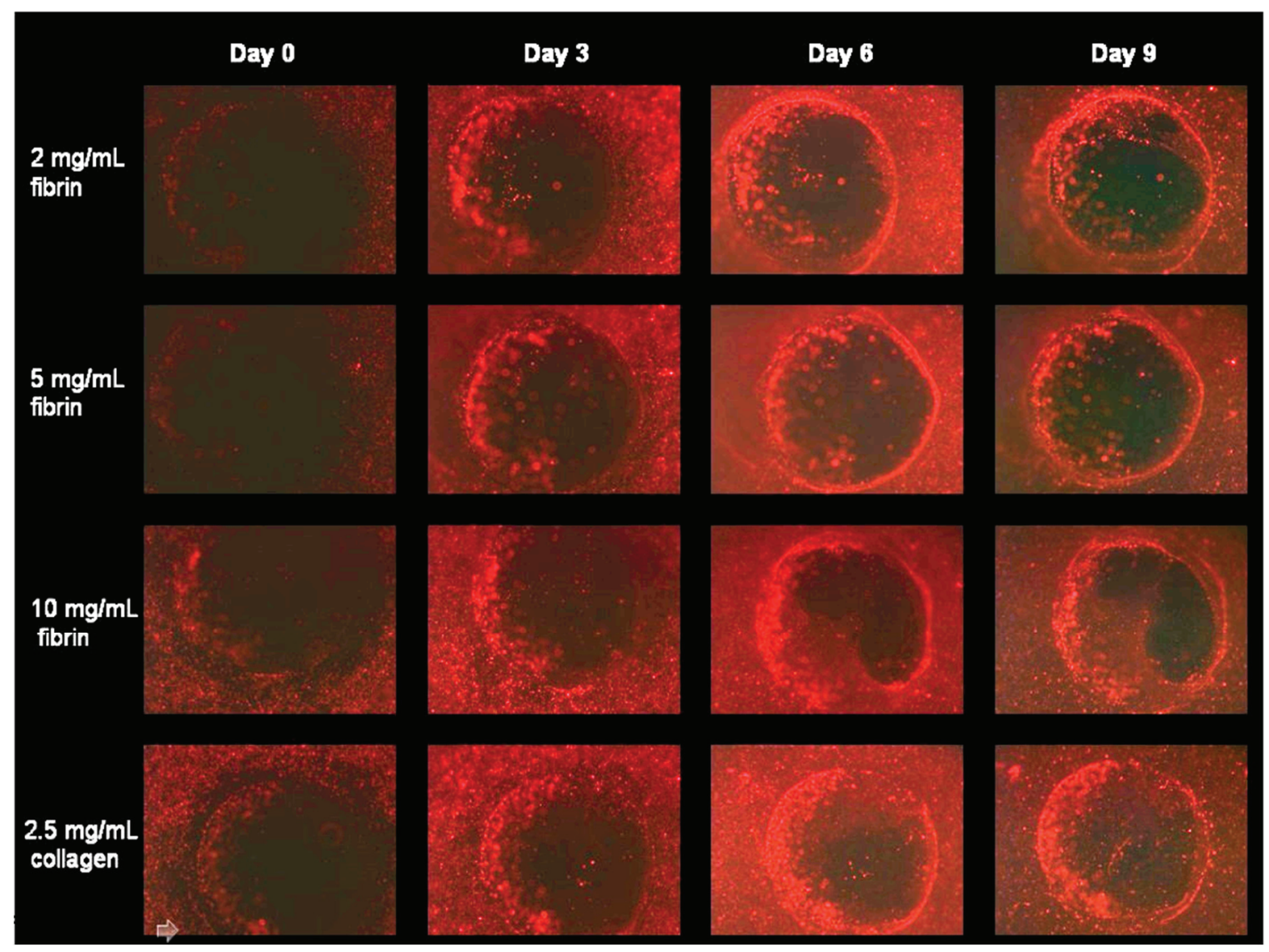

\section{Discussion}

A better understanding of the wound healing process leads to generating better products that aid in healing hard-to-heal wounds such as chronic wounds. Cell migration during the wound healing process is an important step of a complete healing. In vivo, the wound healing process includes different cell types (macrophages, fibroblasts, and keratinocytes) coming in and out of the wound bed at different time points. Mimicking the in vivo milieu with an in vitro 3D model will assist wound healing scientists to better understand the wound healing process and aid companies in generating better products that heal wounds faster.

Existing 3D wound healing models have advantages and disadvantages. For example, a model established by Karamichos et al. [12] uses 3D collagen matrices whereby cells migrate out of the matrix. The limitation of this model is the use of only one cell type. Another 3D in vitro model is "human skin equivalents" which consist of a stratified layer of keratinocytes and keep the surface at an air-liquid interface. However, again this model only introduces one cell type [9]. Finally, there is a 3D in vivo model where skin equivalents are cultured with keratinocytes and then grafted into mice [8]. Our novel $3 \mathrm{D}$ in vitro model allows for the study of cell migration between two different substrates and the use of many different cell types, more closely resembling the milieu of the wound environment.

In this study, we have established a novel wound healing model that is more representative of the in vivo wound than the existing 3D models. We have examined fibroblasts' migration from collagen into 
a collagen-filled "wound" and from collagen into a fibrin-filled "wound." We found that fibroblasts migrated over half of the collagen wound by day 3, and completely covered the defect by day 6 in small defects. Fibroblast migration rate from 3D collagen into collagen defects was similar for both small and large defects and was equal to $249.09 \mu \mathrm{m} /$ day. We have also found that taking a condition medium from a 2D scratched wound healing increased the migration rate slightly but not statistically significantly (Figures 4 and 5) over untreated 3D collagen models, suggesting that some factors released from scratched wounds might be affecting cell migration. Ongoing work is focusing on identifying these factors. Furthermore, we found that fibroblasts' migration rate from collagen to fibrin was $144 \mu \mathrm{m} /$ day on average (Figure 7), which closely resembles the actual wound environment. The distance of cell migration observed from the collagen to fibrin model is less than those from the collagen-to-collagen model, but closer to the in vivo studies.

There is a need for a more complex model than the 2D scratch wound assay to study migration in the wound environment. Cultured skin equivalents grafted into mice resulted in a cellular migration rate of $120 \pm 75 \mu \mathrm{m} /$ day. The average migration rate calculated in our collagen-to-collagen wound model was $249.09 \mu \mathrm{m} /$ day. On the other hand, the average migration rate in the collagen-to-fibrin model, which is more representative of the wound environment, was $144 \mu \mathrm{m} /$ day, which is similar to the range found in the in vivo study. This demonstrates that our model closely represents the wound environment.

We are in the process of modifying the model further. For example, we are in the process of adding monocytes to create a baseline of how growth factors secreted from monocytes that are typically present in the wound healing environment affect fibroblast and keratinocyte migration (Figure 1). We are also studying the effect of localized release of various growth factors such as KGF, FGF, TGF- $\beta$, PDGF, and CTACK/CCL27 on cell migration. This localized release is accomplished by creating fibrin beads with growth factors and embedding these beads inside the fibrin defects. The growth factors are diffused outwards, allowing us to observe their effect on the migration of fibroblasts and keratinocytes. A 3D construct that contains monocytes, fibroblasts, stratified keratinocytes, and fibrin beads with growth factors embedded is a more complex model to study wound healing.

Figure 1. Representation of 3D migration assay.

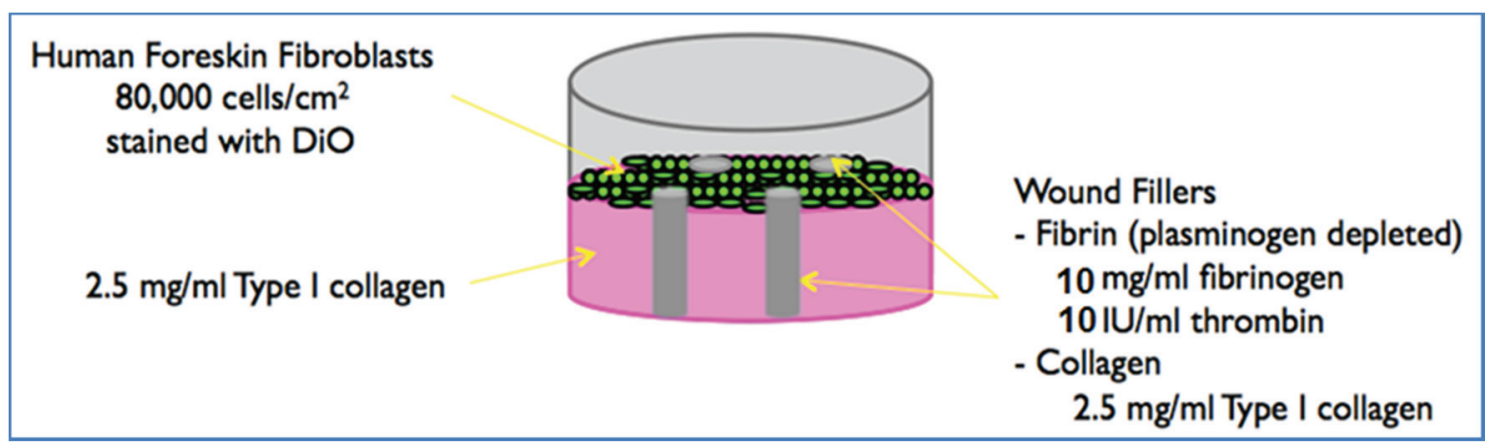

\section{Conclusion}

We present here a novel 3D wound healing model by introducing a defect filled with fibrin in a 3D collagen construct. The model is used to determine the effects of various growth factors, delivered in the wound defects, on fibroblasts' and keratinocytes' migration into the defects. This novel 3D wound healing model offers a complex wound healing assay than existing wound models. 


\section{Acknowledgements}

The authors thank Haison Duong and Chase Linsley for technical assistance and conceptual input.

\section{Author Contributions}

The first two authors execute the experiments equally.

\section{Conflicts of Interest}

The authors declare no conflict of interest

\section{References}

1. Brown, G.L.; Nanney, L.B.; Griffen, J.; Cramer, A.B.; Yancey, J.M.; Curtsinger, L.J., 3rd; Holtzin, L.; Schultz, G.S.; Jurkiewicz, M.J.; Lynch, J.B. Enhancement of wound healing by topical treatment with epidermal growth factor. N. Engl. J. Med. 1989, 321, 76-79.

2. Brown, L.F. Berse, B.; Yeo, T.K.; Senger, D.R.; Dvorak, H.F.; van de Water, L. Expression of vascular permeability factor (vascular endothelial growth factor) by epidermal keratinocytes during wound healing. J. Exp. Med. 1992, 176, 1375-1379.

3. Carlson, M.W.; Dong, S.; Garlick, J.A.; Egles, C. Tissue-engineered models for the study of cutaneous wound-healing. In Studies in Mechanobiology, Tissue Engineering and Biomaterials; Gefen, A., Ed.; Springer: New York, NY, USA, 2009; Volume 1, pp. 263-280.

4. Catelas, I.; Sese, N.; Wu, B.; Dunn, J.; Helgerson, S.; Tawil, B. Human mesenchymal stem cells proliferation and osteogenic differentiation in fibrin gels in vitro. Tissue Eng. 2006, 12, 2385-2396.

5. Chiu, C.; Hecht, V.; Duong, H.; Wu, B.; Tawil, B. Permeability of three-dimensional fibrin constructs corresponds to fibrinogen and thrombin concentrations. BioRes. 2012, 1, 34-40.

6. Clark, R.A.F. Cuntaneous wound healing. N. Engl. J. Med. 1999, 341, 738-746.

7. Cox, S.; Cole, M.; Tawil, N. Behavior of human dermal fibroblasts in 3 dimensional fibrin clots: Dependence on the fibrinogen and thrombin concentration. Tissue Eng. 2004, 10, 942-954.

8. Duong, H.; Wu, B.; Tawil, B. Modulation of 3-dimensional fibrin matrix stiffness by intrinsic fibrinogen-thrombin compositions and by extrinsic cellular activity. Tissue Eng. 2009, 15, 1865-1876.

9. Geer, D.; Swartz, D.; Andreadis, S. In vivo model of wound healing based on transplanted tissue-engineered skin. Tissue Eng. 2004, 10, 1006-1017.

10. Hebda, P.A.; Klingbeil, C.K.; Abraham, J.A.; Fiddes, J.C. Basic fibroblast growth factor stimulation of epidermal wound healing in pigs. J. Invest. Dermatol. 1990, 95, 626-631.

11. Ho, W.; Tawil, B.; Dunn, J.C.Y.; Wu, B.M. The behavior of human mesenchymal stem cells in 3D fibrin clots: Dependence on fibrinogen concentration and clot structure. Tissue Eng. 2006, 12, 1587-1595.

12. Karamichos, D.; Lakshman, N.; Petroll, M. An experimental model for assessing fibroblast migration in 3-D collagen matrices. Cell Motil. Cytoskel. 2009, 66, 1-9.

13. Linsley, C.; Wu, B.; Tawil, B. The effect of fibrinogen, collagen type I and fibronectin on mesenchymal stem cells growth and differentiation into osteoblasts. Tissue Eng. 2013, 19, $1416-1423$. 
14. Lynch, S.E.; Robert, B.C.; Harry, N.A. Growth factors in wound healing. Single and synergistic effects on partial thickness porcine skin wounds. J. Clin. Invest. 1989, 84, 640-646.

15. Mana, M.; Cole, M.; Cox, S.; Tawil, B. Human U937 monocyte behavior and protein expression on various formulations of three-dimensional fibrin clots. Wound Repair Regen. 2006, 14, 72-80.

16. Mooney, R.; Tawil, B.; Mahoney, M. Specific fibrinogen and thrombin concentrations promote neuronal rather than glial growth when primary neural cells are seeded within plasma-derived fibrin gels. Tissue Eng. 2010, 16, 1607-1619.

17. Puchtler, H.; Waldrop, F.S.; Valentine, L.S. Polarization microscopic studies of connective tissue stained with picrosirius red FBA. Beitr. Pathol. 1973, 150, 174-187.

18. Rich, L.; Whittaker, P. Collagen and picrosirius red staining: A polarized light assessment of fibrillar hue and sptail distribution. Braz. J. morphol. Sci. 2005, 22, 97-104.

19. Sese, N.; Cole, M.; Tawil, B. Cross-talk between human dermal fibroblasts and keratinocytes co-cultured in 3 dimensional fibrin clots. Tissue Eng. 2011, 17, 429-437.

20. Tao, H.; Berno, A.; Cox, D.; Frazer, K. In vitro human keratinocyte migration rates are associated with SNPs in the KRT1 interval. PLOS ONE 2007, 2, e697.

21. Tawil, B.; Wu, B. Three-dimensional fibrin constructs in tissue engineering. Review. In An Introduction to Biomaterials, 2nd ed.; Hollinger, J.O., Ed.; CRC Taylor \& Francis: New York, NY, USA, 2012; pp. 249-262.

22. Xie, Y.; Rizzi, S.C.; Dawson, R.; Lynam, E.; Richards, S.; Leavesley, D.I.; Upton, Z. Development of a three-dimensional human skin equivalent wound model for investigating novel wound healing therapies. Tissue Eng. Part C 2010, 16, 1111-1123.

(C) 2014 by the authors; licensee MDPI, Basel, Switzerland. This article is an open access article distributed under the terms and conditions of the Creative Commons Attribution license (http://creativecommons.org/licenses/by/4.0/). 